\title{
Spectrum of transthyretin gene mutations and clinical characteristics of Polish patients with cardiac transthyretin amyloidosis
}

\author{
Monika Gawor ${ }^{1}$, Katarzyna Holcman ${ }^{2}$, Maria Franaszczyk ${ }^{3}$, Marta Lipowska ${ }^{4}$, \\ Piotr Michałek ${ }^{5}$, Anna Teresińska ${ }^{6}$, Zofia T. Bilińska ${ }^{7}$, Paweł Rubiśs ${ }^{2}$, \\ Magdalena Kostkiewicz ${ }^{2,8}$, Wojciech Szot ${ }^{8}$, Piotr Podolec ${ }^{2}$, Jacek Grzybowski ${ }^{1}$ \\ ${ }^{1}$ Department of Cardiomyopathy, Institute of Cardiology, Warsaw, Poland \\ ${ }^{2}$ Department of Cardiac and Vascular Diseases, John Paul II Hospital, \\ Jagiellonian University Medical College, Krakow, Poland \\ ${ }^{3}$ Department of Medical Biology, Institute of Cardiology, Warsaw, Poland \\ ${ }^{4}$ Department of Neurology, Medical University of Warsaw, Poland \\ ${ }^{5}$ Rapid Diagnosis Department, Emergency Room, Institute of Cardiology, Warsaw, Poland \\ ${ }^{6}$ Department of Nuclear Medicine, Institute of Cardiology, Warsaw, Poland \\ ${ }^{7}$ Unit for Screening Studies in Inherited Cardiovascular Diseases, \\ The Cardinal Stefan Wyszynski Institute of Cardiology, Warsaw, Poland \\ ${ }^{8}$ Department of Nuclear Medicine, John Paul II Hospital, \\ Jagiellonian University Medical College, Krakow, Poland
}

\begin{abstract}
Background: Transthyretin amyloidosis (ATTR) is a rare, life-threatening systemic disorder. We present first findings on the cardiac hereditary ATTR in Poland.

Methods: Sixty-eight consecutive patients with suspected or known cardiac amyloidosis were evaluated, including blood tests, standard 12-lead electrocardiography (ECG) and transthoracic echocardiography. ATTR was confirmed histologically or non-invasively using $99 m T c-D P D$ scintigraphy. Transthyretin (TTR) gene sequencing was performed.

Results: In 2017-2019, 10 unrelated male patients were diagnosed with hereditary ATTR. All patients had very uncommon TTR gene mutations: 7 patients had p.Phe53Leu mutation, 2 patients had p.Glu109Lys mutation and 1 patient had p.Ala101Val mutation. The age of onset ranged from 49 to 67 years (mean [SD] age, 58.7 [6.4] years). On ECG, most patients (70\%) had pseudoinfarct pattern and/or low QRS voltage. The maximal wall thickness (MWT) on echocardiography varied considerably among the patients from moderate $(16 \mathrm{~mm})$ to massively increased $(30 \mathrm{~mm})$. Most patients $(90 \%)$ had decreased left ventricular ejection fraction (mean [SD], 43 [11] \%). On follow-up, we observed progressive heart failure in almost all cases. The first patient with p.Phe53Leu mutation died of heart failure, the second died suddenly, the third successfully underwent combined heart and liver transplant with 15 months survival from the surgery. The patient with p.Ala101Val mutation died of stroke.

Conclusions: According to available data, this is the first time that the types of TTR mutations and the clinical characteristics of Polish patients with cardiac hereditary ATTR have been described. Previous literature data about Polish background in families with p.Phe53Leu mutation and the present results, suggest that this TTR mutation might be endemic in the Polish population. (Cardiol J 2022; 29, 6: 985-993)
\end{abstract}

Key words: cardiac amyloidosis, hereditary transthyretin amyloidosis, transthyretin amyloidosis, transthyretin cardiomyopathy, transthyretin mutation

Address for correspondence: Monika Gawor, MD, PhD, Department of Cardiomyopathy, Institute of Cardiology, ul. Alpejska 42, 04-628 Warszawa, Poland, tel: +48 2234346 71, e-mail: mgawor@ikard.pl

Received: 19.12.2019 Accepted: 13.07.2020 Early publication date: 11.08.2020

This article is available in open access under Creative Common Attribution-Non-Commercial-No Derivatives 4.0 International (CC BY-NC-ND 4.0) license, allowing to download articles and share them with others as long as they credit the authors and the publisher, but without permission to change them in any way or use them commercially. 


\section{Introduction}

Transthyretin amyloidosis (ATTR) is a rare, life-threatening systemic disorder. It results from extracellular deposition of transthyretin (TTR)-derived amyloid fibrils leading to dysfunction of affected organs. It occurs in both a wild-type form (wtATTR) and a hereditary form (hATTR) transmitted in an autosomal dominant inheritance with a variable penetrance [1]. Without treatment the disease progresses gradually. According to recent reports, median survival of patients with cardiac ATTR is approximately 25 months to 41 months from onset of the first symptoms. The prognosis is worse in cases of hATTR [2].

More than 140 different disease-causing mutations of the TTR gene have been reported [3]. The prevalence of different mutations varies according to ethnicity and geographic region. Although there are endemic regions of ATTR such as Portugal, Sweden and Japan with certain high frequency mutations, the disease has been identified worldwide $[1,4-6]$.

Transthyretin amyloidosis is known for its heterogeneity. Some TTR mutations induce cardiomyopathy as a main presentation while others are associated primarily with familial amyloid polyneuropathy. Other manifestations of hATTR often include gastrointestinal, renal and ocular symptoms as well as carpal tunnel syndrome $[2,7,8]$. The phenotypic expression can vary not only among mutations but within mutations. The geographical origin and ethnic background of the patient can have implications for disease onset and initial symptoms for a given mutation [1]. Surprisingly, cases of monozygotic twins, in which one twin develops the disease and the other does not, have also been reported, proving that there are factors modulating amyloid fibrils formation $[9,10]$.

Screening of the TTR gene and differentiation hATTR from wtATTR have serious clinical implications. In contrast to wtATTR, patients with hATTR may benefit from liver transplant or combined heart and liver transplant. Information on the type of $T T R$ mutation provides prognostic value not only for the proband but also for his family members. The spectrum of TTR gene mutations in Polish patients with cardiac ATTR and their clinical characteristics have not yet been described. Therefore, this study presents for the first time the results of genetic testing and a description of clinical heterogeneity of patients with cardiac ATTR diagnosed in our centers during the last 3 years.

\section{Methods}

The study complies with the principles outlined in the Declaration of Helsinki and was approved by the Institutional Ethics Committee.

\section{Study population}

The study included consecutive patients diagnosed with hereditary cardiac ATTR in 2017-2019. 68 consecutive patients examined with suspected or known cardiac amyloidosis were referred to two cardiology centers for further evaluation. All patients underwent routine assessment including medical history, physical examination, blood tests with $\mathrm{N}$-terminal pro-B-type natriuretic peptide (NT-proBNP) and high-sensitive cardiac troponin T (hs-cTnT) measurements, as well as standard 12-lead electrocardiography (ECG) and transthoracic echocardiography. ATTR was confirmed histologically with immunohistochemistry and/or non-invasively (intensive cardiac uptake at $99 \mathrm{mTc}-\mathrm{DPD}$ bone scintigraphy) [11]. Light chain amyloidosis was excluded by free light chain testing: conventional electrophoresis and immunofixation of serum or urine. Patients underwent $T T R$ gene sequencing as part of their routine workup. Neurological assessment was performed in all patients with hATTR. Informed written consent was obtained from each participant included in the study.

\section{Analysis of TTR gene}

Patients assessed at the Institute of Cardiology, Warsaw, Poland underwent genetic testing in a local laboratory. Genomic DNA in all patients was extracted from whole blood leukocytes with the salting-out method. The entire coding regions of the TTR gene together with splice sites were sequenced with the Sanger method using a 3130xL Genetic Analyzer (Life Technologies, Foster City, California, United States) and the Big Dye Terminator v1.1 Cycle Sequencing Kit (Life Technologies) according to manufacturer instructions. For chromatograms analysis Variant Reporter 1.1 (Life Technologies) was used. All patients from both centers also underwent commercially available $T T R$ genetic testing developed and performed by Centogene AG, Rostock, Germany.

\section{Results}

Of the 68 patients, $10(14.7 \%)$ male patients had cardiac hATTR, 19 (27.9\%) had wtATTR, $9(13.2 \%)$ were diagnosed with cardiac light chain amyloidosis and $30(44.1 \%)$ had no evidence of 
amyloid deposition. All patients with hATTR had very uncommon TTR gene mutations: p.Phe53Leu (NM_000371.3:c.157T >C, rs121918068), p.Glu109Lys (NM_000371.3:c.325G $>$ A) and p.Ala101Val (NM_000371.3:c.302C > T). The most prevalent was p.Phe53Leu mutation identified in 7 out of 10 patients with hATTR. The baseline clinical data of the patients with hATTR was shown in Table 1.

The age of onset ranged from 49 to 67 years (mean [SD] age, 58.7 [6.4] years) and differed considerably even among patients with the same mutation. Family history suggestive of cardiac amyloidosis was positive in 6 probands. On ECG, most patients $(70 \%)$ had a pseudoinfarct pattern in precordial leads. Similarly, most patients (70\%) had low QRS voltage in limb leads. Echocardiography revealed concentrically increased thickness of left ventricular (LV) wall in all patients. The maximal wall thickness (MWT) varied markedly among the patients from moderate $(16 \mathrm{~mm})$ to massively increased $(30 \mathrm{~mm}$ ) (mean [SD] MWT, $22[4] \mathrm{mm})$. Most patients (90\%) had decreased LV ejection fraction (LVEF) (mean [SD] LVEF, 43 [11] \%). Baseline laboratory examination revealed increased levels of hs-cTnT and NT-proBNP in all patients. Even individuals with initially mild symptoms of heart failure (HF) had elevated serum concentrations of examined biomarkers (Fig. 1).

Seven patients presented with mixed-phenotype and suffered also from polyneuropathy. Only 2 patients primarily presented with polineuropathy (patients with p.Phe53Leu mutation) and cardiac assessment was performed after familial amyloid polyneuropathy was confirmed. Five other patients were diagnosed with polyneuropathy during followup. Of the 10 patients, 7 suffered from gastrointestinal disturbances. However, it was difficult for the patients to determine at what stage of the disease the first symptoms from the gastrointestinal tract appeared and whether they preceded the appearance of cardiac manifestations. Five patients presented with carpal tunnel syndrome, the so-called 'red flag' symptom for ATTR, which evidently preceded the appearance of cardiac manifestations. One of the patients had ocular symptoms, which seem to occur frequently in patients with p.Glu109Lys mutation. Orthostatic hypotension was present in 4 patients with advanced symptoms of HF regardless of the presence of polyneuropathy.

\section{Clinical characteristics of patients with p.Phe53Leu TTR mutation}

Although the patients with p.Phe53Leu TTR mutation had heterogeneous clinical presentation and were diagnosed at various stages of the disease, ranging from accidentally discovered disease in asymptomatic patient to the advanced stages of biventricular HF, all of them had significant abnormalities on ECG and echocardiography from the first assessment. It is noteworthy that all patients except for proband 6 , who was diagnosed at a very early stage of the disease, had reduced LV systolic function. Interestingly, symptoms of HF assessed in accordance to the New York Heart Association (NYHA) classification, did not reflect the impairment of LV systolic function - patients with only mild symptoms of HF had already significantly impaired LVEF (Fig. 2). Most of the patients with p.Phe53Leu mutation (71.4\%), except for 2 cases, presented with mixed phenotype and had also polyneuropathy at various stages of advancement.

The case of proband 3 who was diagnosed with hATTR at a time when he was completely asymptomatic with confirmed good exercise tolerance, is particularly interesting. He was referred for cardiological assessment due to ECG abnormalities found during annual medical check-up (sinus rhythm with low QRS voltage in the limb leads and nonspecific ST-T wave changes). Physical examination and chest X-ray were normal. However, baseline levels of NT-proBNP and hs-cTnT were significantly increased from the beginning. Echocardiography revealed moderately increased LV wall thickness (MWT $16 \mathrm{~mm}$ ), mildly enlarged left atrium and significantly reduced LV systolic function without segmental hypokinesis (LVEF 40\%). Moreover, coronary computed tomography angiography, performed to reveal the reasons for the impaired LVEF, showed right coronary artery (RCA) chronic total occlusion as well as stenosis in the left main coronary artery, in the left anterior descending artery (LAD) and in the left marginal artery. The patient was diagnosed with occult multivessel coronary artery disease. Invasive coronary angiography with fractional flow reserve demonstrated that only stenosis in the LAD was hemodynamically relevant. Percutaneous coronary intervention using drug eluting stents was performed for occlusion in the RCA and for stenosis in LAD. Although at 20-month follow-up, the patient remained asymptomatic, with good exercise tolerance confirmed on ECG exercise testing, further deterioration of LV function and a significant increase of cardiac biomarkers were observed. HF symptoms progressed rapidly, however signs of polyneuropathy never appeared in this patient. Ultimately, the patient underwent successful combined heart and liver transplantation. 


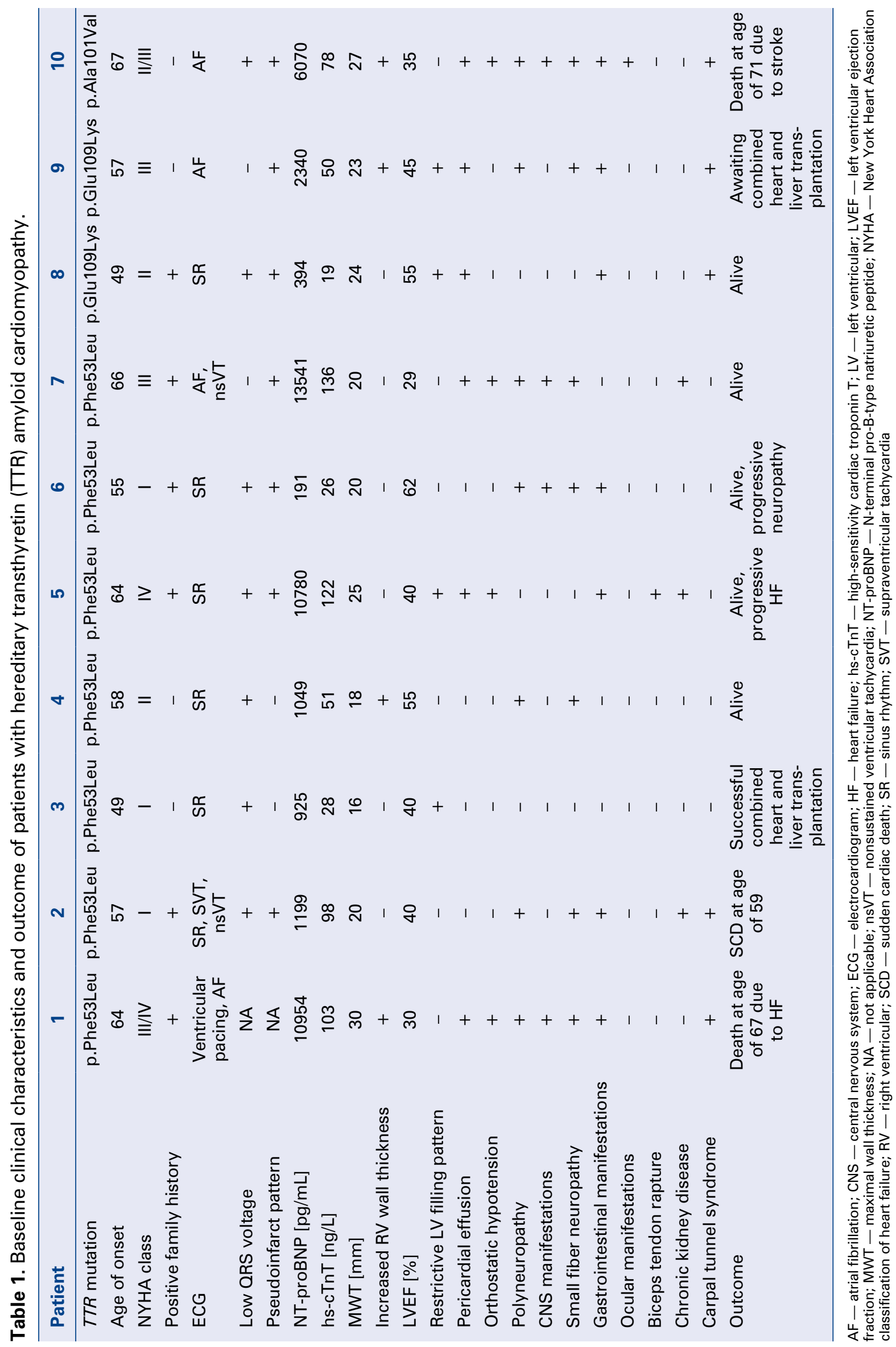




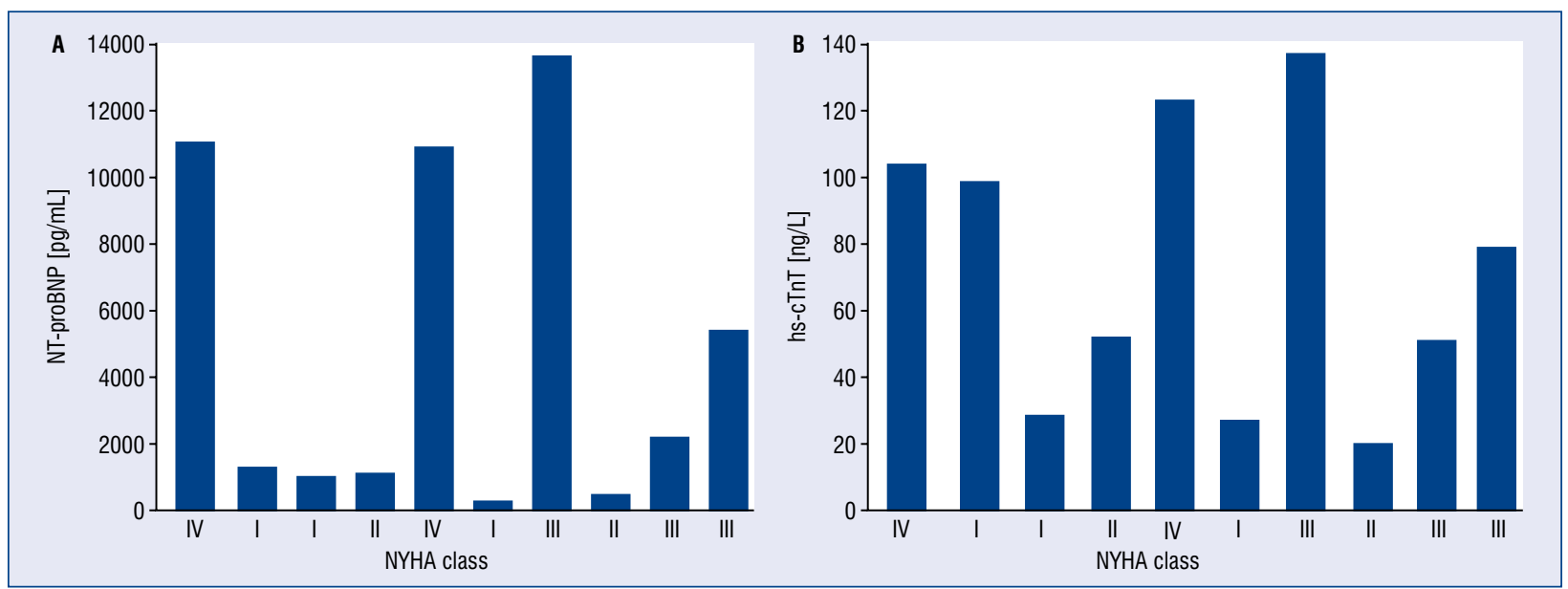

Figure 1. Baseline $\mathrm{N}$-terminal pro-B-type natriuretic peptide (NT-proBNP; normal range: 0-125 pg/mL, A) and high-sensitive cardiac troponin T (hs-cTnT; normal range: $0-14 \mathrm{ng} / \mathrm{L}, \mathrm{B}$ ) levels in the patients classified according to the New York Heart Association (NYHA) classification of heart failure.

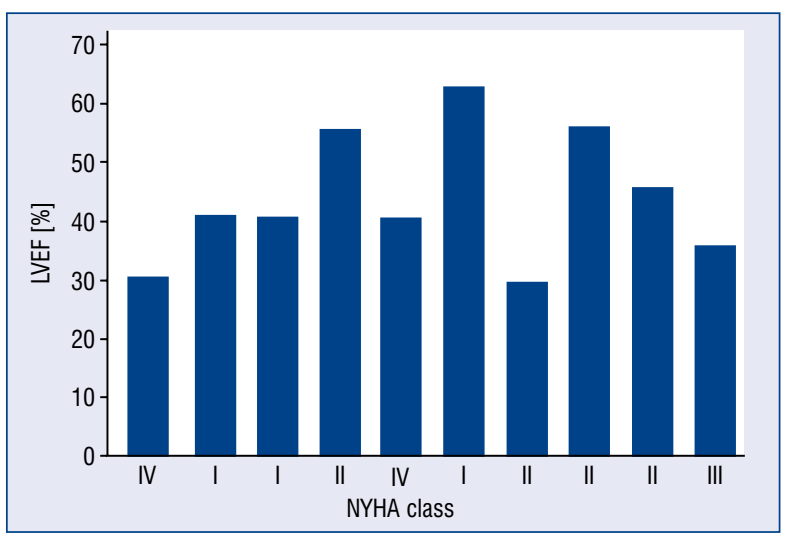

Figure 2. Left ventricular ejection fraction (LVEF) in the patients classified according to the New York Heart Association (NYHA) classification of heart failure.

Interestingly, proband 1, a 64-year-old male with advanced biventricular HF, also had a history of ischemic heart disease treated with coronary artery bypass graft surgery. The patient had many comorbidities, including atrial fibrillation (AF) with third--degree atrioventricular block requiring pacemaker implantation, subsequently upgraded to cardiac resynchronization therapy, polyneuropathy grade 1 (he was able to walk without aid), recurrent pleural effusion and bilateral carpal tunnel syndrome.

\section{Clinical characteristics of patients with p.Glu109Lys TTR mutation}

Two patients with features of restrictive cardiomyopathy and carpal tunnel syndrome were identified with the p.Glu109Lys TTR mutation. Proband 8, a 49-year-old male, was diagnosed at a very early stage of the disease (NYHA class I), without overt signs of HF, with only mildly increased levels of cardiac serum biomarkers. However, echocardiography and ECG demonstrated significant abnormalities, including mildly reduced LV systolic function, restrictive LV filling pattern, small pericardial effusion as well as pseudoinfarct pattern and low QRS voltage in limb leads. The patient had a family history suggestive of cardiac amyloidosis.

A second patient with p.Glu109Lys TTR mutation, proband 9 , was referred to our institution due to a 3 month history of progressive exertional dyspnea (NYHA class III at initial presentation) and massive lower-limbs edema. He also suffered from periorbital purpura, weight loss, mild symptoms of polyneuropathy (predominantly paresthesias) and ocular symptoms including vitreous opacities. His family history was unremarkable. ECG demonstrated occult AF of unknown duration, pseudoinfarct pattern, nonspecific ST segment and T-wave abnormalities. QRS voltage was in the lower range of the norm. The chest X-ray showed pulmonary congestion and small left sided pleural effusion. Echocardiography revealed increased LV wall thickness (max. $23 \mathrm{~mm}$ at interventricular septum), sparkling echoes, restrictive LV filling pattern and decreased LVEF $45 \%$. Right ventricle (RV) was enlarged with increased wall thickness. Both atria were augmented and small pericardial effusion was present. Diagnosis of ATTR was 


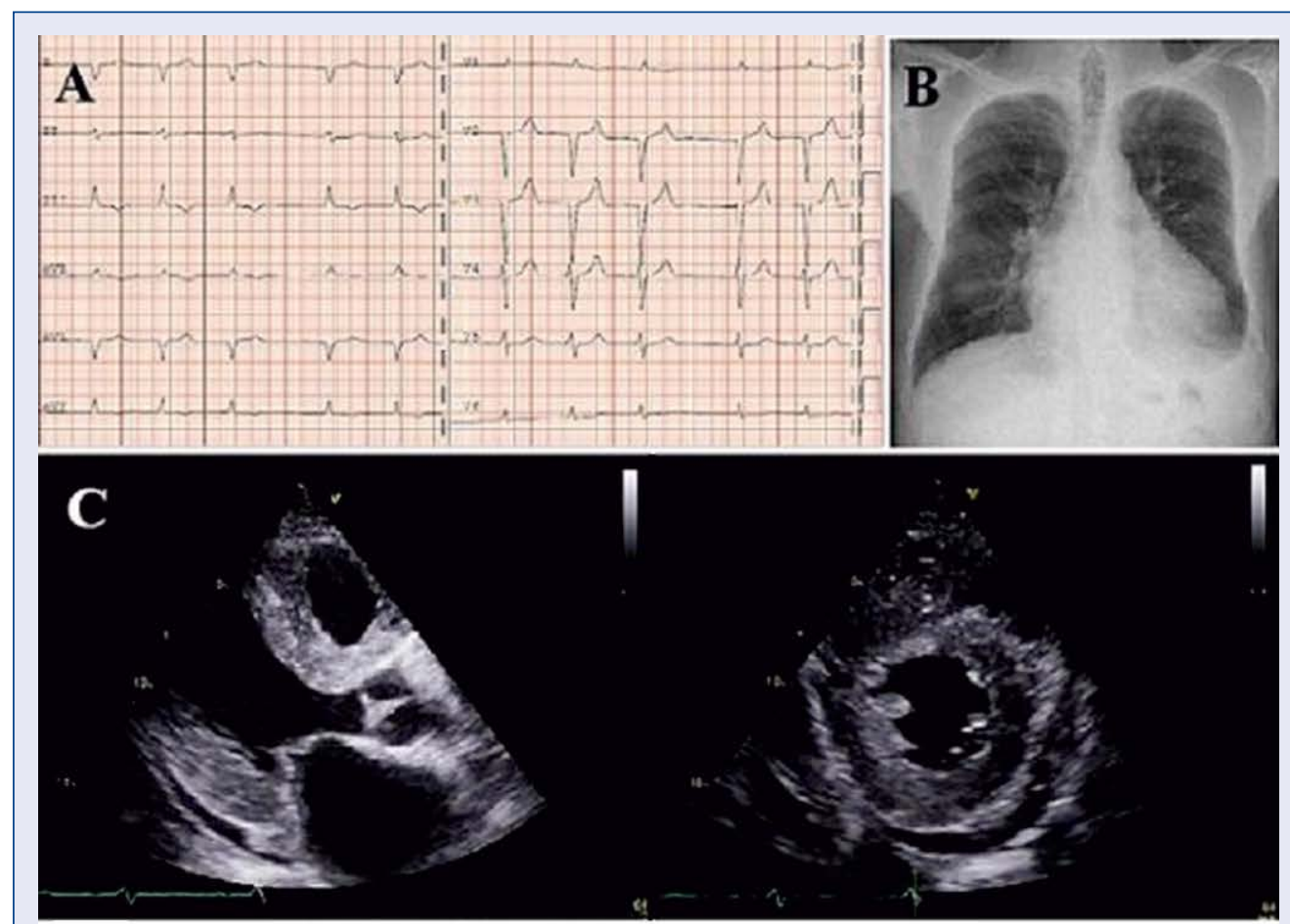

D

Figure 3. Clinical data of proband 9; A. Standard 12-lead electrocardiogram; B. Chest X-ray; C. Transthoracic echocardiography; D. Bone scintigraphy with $99 \mathrm{mTc}-\mathrm{DPD}$ tracer confirming transthyretin amyloid cardiomyopathy in anterior (left) and posterior (right) whole body scan.

confirmed with bone scintigraphy with $99 \mathrm{~m}-\mathrm{DPD}$ tracer (Fig. 3A-D). Two months after initial presentation the patient was stable but with a further increase in NT-proBNP and hs-cTnT levels (3513 $\mathrm{pg} / \mathrm{mL}$ and $68 \mathrm{ng} / \mathrm{L}$, respectively). He was referred for compassionate treatment with tafamidis as a bridge to combined heart and liver transplantation.

\section{Clinical characteristics of patients with p.Ala101 Val TTR mutation}

Only 1 patient, 67-year-old male with progressive HF (NYHA class II/III at initial presentation), permanent AF, significantly increased levels of cardiac biomarkers was identified with p.Ala101Val $T T R$ mutation. Echocardiography of this patient 
showed massively increased RV and LV wall thickness (max. $27 \mathrm{~mm}$ at interventricular septum), significantly decreased LV systolic function (LVEF 35\%) and small pericardial effusion. The patient also suffered from orthostatic hypotension, bilateral carpal tunnel syndrome, mild symptoms of polyneuropathy and monoclonal gammapathy of unknown significance. Due to the presence of only microdeposits of amyloid in the tissue biopsy and no access to $99 \mathrm{mTc}-\mathrm{DPD}$ scintigraphy at that time, diagnosis of ATTR in the patient was established using mass spectrometry.

\section{Follow-up}

Information about the outcome of patients with hATTR are collected in Table 1 . In almost all cases progressive HF was observed. One patient with p.Phe53Leu TTR mutation (proband 1) died of biventricular HF, proband 2 died due to sudden cardiac death, proband 3 successfully underwent combined heart and liver transplantation with a 15-month survival from the surgery. One patient with p.Glu109Lys $T T R$ mutation, proband 9, is currently waiting for combined heart and liver transplantation. Patient with p.Ala101Val TTR mutation (proband 10) died of stroke.

\section{Discussion}

Herein, we report for the first time the types of TTR mutations and the clinical characteristics of Polish patients with cardiac hATTR. Currently, literature data on Polish patients with cardiac amyloidosis concerns mainly light chain amyloidosis and consists of only a few case reports and two original papers [11-15]. The presented study, although based on the small cohort of patients from two cardiology centers, fills an important gap in diagnosis and clinical management of cardiac ATTR in Poland. This research highlights that improvements in diagnostics, in particular introducing genetic analysis of the TTR gene and $99 \mathrm{mTc}-\mathrm{DPD}$ scintigraphy into a routine diagnostic path of patients with suspicion of ATTR, hugely facilitates diagnostics. Moreover, it allows to perform a non-invasive diagnosis $[8,16]$. It is crucial to identify patients with ATTR, especially now, when the targeted treatment of ATTR is possible.

The present study reports patients with unexpectedly rare $T T R$ mutations: p.Phe53Leu, p.Ala101Val and p.Glu109Lys. Surprisingly, none of the most prevalent TTR variants: p.Val50Met, p.Val142Ile or p.Thr80Ala, were identified $[4,17$, 18]. Moreover, a high incidence of p.Phe53Leu
$T T R$ mutation were observed. This occurred in the majority of the patients $(70 \%)$.

The phenylalanine 53 is highly conserved amino acid of the TTR protein across species [19]. Position 53 of TTR protein is not directly involved in the formation of the transthyretin tetramer, however, loss of an aromatic ring associated with phenylalanine, may change the molecule conformation and lead to fibril formation [20]. The p.Phe53Leu $T T R$ mutation was described previously only in 7 unrelated families including 2 American families of Polish descent and single Swedish, Taiwanese, Chinese, Hungarian and Israeli families [19-25]. This mutation was associated mostly with lateonset, progressive polyneuropathy and simultaneous presentation of severe cardiomyopathy. Previous literature data about a Polish background in families with p.Phe53Leu mutation and our findings (another 7 unrelated Polish families identified with p.Phe53Leu mutation) imply that this $T T R$ mutation might be very frequent in the Polish population [20,21]. Of note, almost all of the patients with p.Phe53Leu mutation presented in this study $(85.7 \%)$ were of south Poland origin, suggesting that this might be an endemic region, where p.Phe53Leu mutation is found with high frequency. It is also possible that this rare mutation was introduced into the population by a common ancestor. However, based on available data it is not possible to prove so-called "founder effect". The heterogeneous clinical presentation of the patients with p.Phe53Leu TTR mutation (different age of onset, concomitant polyneuropathy but not in all cases) suggests also that other genetic or environmental factors may be involved in the disease development and progression.

p.Glu109Lys TTR mutation was reported previously only in 3 families and is characterized by early onset, concomitant ocular manifestation and severe phenotype with early heart dysfunction leading to heart and liver transplantation [26-28]. A similar course of the disease was observed in the case of proband 9 , including the rapid progression of $\mathrm{HF}$ and ocular manifestations.

p.Ala101Val TTR mutation is an unusually rare mutation. So far, only 2 patients were previously reported with this mutation, including 1 patient from Institute of Cardiology who was diagnosed genetically in the National Amyloidosis Center in London in 2008 [17]. The patient suffered from severe biventricular HF with massive leg edema, persistent pericardial and pleural effusion requiring recurrent hospitalizations. ECG demonstrated sinus rhythm, first degree atrioventricular block 
(PR $320 \mathrm{~ms}$ ), low QRS voltage in limb leads and regression of $r$ waves in lateral (V5-V6) leads. Echocardiography demonstrated features of restrictive cardiomyopathy with concentrically increased LV wall thickness (max. $15 \mathrm{~mm}$ ), moderated left atrium enlargement and mildly decreased LV systolic function (LVEF 50\%). During follow-up, further deterioration of LV function was observed (LVEF $38 \%$ ) as well as features of moderate pulmonary hypertension (PASP $40 \mathrm{mmHg}$ ), thickening of RV wall and dilatation of both atria. The patient died of advanced $\mathrm{HF}$ while awaiting heart transplantation.

In the present study, in line with the previous literature data, a high sensitivity of cardiac biomarkers for diagnostic and prognostic purposes was observed [29]. Even patients with only mild symptoms of HF (NYHA I) or asymptomatic patients, had already elevated serum levels of cardiac biomarkers (Fig. 1).

\section{Conclusions}

The high prevalence of p.Phe53Leu TTR mutation in the study group and in previous literature data suggest that a possible endemic mutation has been found among Polish patients with cardiac hATTR. Considering the fact that the diagnosis of cardiac hATTR has clinical implications and information on the presence of TTR mutation is useful for affected patients, pre-symptomatic carriers and their relatives, we should identify patients with this disorder and better define the disease. The emergence of novel treatments in cardiac ATTR, which may improve patient prognosis is another argument for the intensification of epidemiological and cross-sectional studies like this.

\section{Acknowledgments}

We would like to acknowledge Centogene AG, Rostock, Germany for support in genetic testing.

\section{Conflict of interest: None declared}

\section{References}

1. Ruberg FL, Grogan M, Hanna M, et al. Transthyretin amyloid cardiomyopathy: JACC state-of-the-art review. J Am Coll Cardiol. 2019; 73(22): 2872-2891, doi: 10.1016/j.jacc.2019.04.003, indexed in Pubmed: 31171094.

2. Castaño A, Drachman BM, Judge D, et al. Natural history and therapy of TTR-cardiac amyloidosis: emerging disease-modifying therapies from organ transplantation to stabilizer and silencer drugs. Heart Fail Rev. 2015; 20(2): 163-178, doi: 10.1007/ s10741-014-9462-7, indexed in Pubmed: 25408161.
3. http://amyloidosismutations.com/mut-attr.php (last accessed December 13th 2019).

4. Ammirati E, AbouEzzeddine OF. Transthyretin amyloidosis in Western Europe: a snapshot from the THAOS registry and a call for further perspectives. Eur Heart J. 2019 [Epub ahead of print], doi: 10.1093/eurheartj/ehz205, indexed in Pubmed: 31006018.

5. Cruz MW, Barroso F, González-Duarte A, et al. The demographic, genetic, and clinical characteristics of Latin American subjects enrolled in the Transthyretin Amyloidosis Outcomes Survey. Amyloid. 2017; 24(sup1): 107-108, doi: 10.1080/13506129.2017.1292239, indexed in Pubmed: 28434322.

6. Maurer M, Hanna M, Grogan M, et al. Genotype and Phenotype of Transthyretin Cardiac Amyloidosis. J Am Coll Cardiol. 2016; 68(2): 161-172, doi: 10.1016/j.jacc.2016.03.596.

7. Witteles RM, Bokhari S, Damy T, et al. Screening for Transthyretin Amyloid Cardiomyopathy in Everyday Practice. JACC Heart Fail. 2019; 7(8): 709-716, doi: 10.1016/j.jchf.2019.04.010, indexed in Pubmed: 31302046.

8. Maurer MS, Bokhari S, Damy T, et al. Expert consensus recommendations for the suspicion and diagnosis of transthyretin cardiac amyloidosis. Circ Heart Fail. 2019; 12(9): e006075, doi: 10.1161/CIRCHEARTFAILURE.119.006075, indexed in Pubmed: 31480867.

9. Holmgren G, Wikström L, Lundgren HE, et al. Discordant penetrance of the trait for familial amyloidotic polyneuropathy in two pairs of monozygotic twins. J Intern Med. 2004; 256(5): 453-456, doi: 10.1111/j.1365-2796.2004.01399.x, indexed in Pubmed: 15485482.

10. Saraiva MJ, Almeida MR, Alves IL, et al. Modulating conformational factors in transthyretin amyloid. Ciba Found Symp. 1996; 199: 47-52, doi: 10.1002/9780470514924.ch4, indexed in Pubmed: 8915603.

11. Szczygieł JA, Wieczorek PZ, Drozd-Sokołowska J, et al. Impaired right ventricular function as a predictor of early mortality in patients with light-chain cardiac amyloidosis assessed in a cardiology department. Pol Arch Intern Med. 2017; 127(12): 854-864, doi: 10.20452/pamw.4135, indexed in Pubmed: 29112180.

12. Prochorec-Sobieszek M, Bilińska ZT, Grzybowski J, et al. Cardiac amyloidosis diagnosed by endomyocardial biopsy. Clinical, histopathological, immunohistochemical and ultrastructural studies. Kardiol Pol. 2005; 63(7): 20-35, indexed in Pubmed: 16136426.

13. Rubiś P, Rudnicka-Sosin L, Jurczyszyn A, et al. The paramount importance of repeated left ventricular endomyocardial biopsy during the diagnosis of restrictive cardiomyopathy due to $\mathrm{AL}$ cardiac amyloidosis. Kardiol Pol. 2016; 74(8): 796, doi: 10.5603/ KP.2016.0114, indexed in Pubmed: 27553348.

14. Gawor M, Mazurkiewicz Ł, Milanowska B, et al. Recovery from heart failure in a patient with cardiac amyloidosis treated with autologous stem cell transplantation. Kardiol Pol. 2017; 75(1): 83, doi: 10.5603/KP.2017.0008, indexed in Pubmed: 28124791.

15. Rajtar-Salwa R, Gębka A, Petkow-Dimitrow P. Non-invasive cardiac imaging methods in transthyretin amyloidosis. Kardiol Pol. 2019; 77(2): 234, doi: 10.5603/KP.2019.0023, indexed in Pubmed: 30816988.

16. Gillmore JD, Maurer MS, Falk RH. Nonbiopsy diagnosis of cardiac transthyretin amyloidosis. Circulation. 2016; 14: 2404-2412, doi: 10.1161/CIRCULATIONAHA.116.021612, indexed in Pubmed: 27143678.

17. Rowczenio D, Quarta CC, Fontana M, et al. Analysis of the TTR gene in the investigation of amyloidosis: A 25-year single UK 
center experience. Hum Mutat. 2019; 40(1): 90-96, doi: 10.1002/ humu.23669, indexed in Pubmed: 30328212.

18. Maurer M, Hanna M, Grogan M, et al. Genotype and Phenotype of Transthyretin Cardiac Amyloidosis. J Am Coll Cardiol. 2016; 68(2): 161-172, doi: 10.1016/j.jacc.2016.03.596.

19. Chen $\mathrm{CH}$, Huang $\mathrm{CW}$, Lee MJ. A case of familial amyloidotic polyneuropathy with a rare Phe33Leu mutation in the TTR gene. J Formos Med Assoc. 2014; 113(8): 575-576, doi: 10.1016/j. jfma.2012.07.026, indexed in Pubmed: 25037766.

20. Harding J, Skare J, Skinner M. A second transthyretin mutation at position 33 (Leu/Phe) associated with familial amyloidotic polyneuropathy. Biochim Biophys Acta. 1991; 1097(3): 183-186, doi: 10.1016/0925-4439(91)90033-6, indexed in Pubmed: 1932142.

21. Myers TJ, Kyle RA, Jacobson DR. Familial amyloid with a transthyretin leucine 33 mutation presenting with ascites. Am J Hematol. 1998; 59(3): 249-251, doi: 10.1002/(sici)10968652(199811)59:3<249::aid-ajh13>3.0.co;2-b, indexed in Pubmed: 9798666.

22. Csillik A, Pozsonyi Z, Soós K, et al. [Transthyretin familial amyloid polyneuropathy - three Hungarian cases with rare mutations (His88Arg and Phe33Leu)]. Ideggyogy Sz. 2016; 69(7-8): 245-253, doi: 10.18071/isz.69.0245, indexed in Pubmed: 29465889.

23. Holmgren G, Hellman U, Jonasson J, et al. A Swedish family with the rare Phe33Leu transthyretin mutation. Amyloid. 2005; 12(3): 189-192, doi: 10.1080/13506120500221989, indexed in Pubmed: 16194875.
24. Leibou L, Frand J, Sadeh M, et al. Clinical and genetic findings in eight Israeli patients with transthyretin-associated familial amyloid polyneuropathy. Isr Med Assoc J. 2012; 14(11): 662-665, indexed in Pubmed: 23240369.

25. Meng LC, Lyu He, Zhang W, et al. Hereditary transthyretin amyloidosis in eight Chinese families. Chin Med J (Engl). 2015; 128(21): 2902-2905, doi: 10.4103/0366-6999.168048, indexed in Pubmed: 26521788.

26. Nakamura M, Hamidi Asl K, Benson MD. A novel variant of transthyretin (Glu89Lys) associated with familial amyloidotic polyneuropathy. Amyloid. 2000; 7(1): 46-50, doi: 10.3109/13506120009146824, indexed in Pubmed: 10842705.

27. Reynolds MM, Veverka KK, Gertz MA, et al. Ocular manifestations of familial transthyretin amyloidosis. Am J Ophthalmol. 2017; 183: 156-162, doi: 10.1016/j.ajo.2017.09.001, indexed in Pubmed: 28911993.

28. Bourque PR, McCurdy AR, Mielniczuk LM, et al. Cardiac amyloidosis phenotype associated with a glu89lys transthyretin mutation. Can J Cardiol. 2017; 33(6): 830.e5-830.e7, doi: 10.1016/j. cjca.2017.01.023, indexed in Pubmed: 28395866.

29. Kristen AV, Maurer MS, Rapezzi C, et al. THAOS investigators. Impact of genotype and phenotype on cardiac biomarkers in patients with transthyretin amyloidosis: Report from the Transthyretin Amyloidosis Outcome Survey (THAOS). PLoS One. 2017; 12(4): e0173086, doi: 10.1371/journal.pone.0173086, indexed in Pubmed: 28384285. 